\title{
Control of Radiation Chemistry during Liquid Cell TEM to Synthesize Transition Metal and Bimetallic Nanoparticles
}

\author{
Taylor J. Woehl
}

University of Maryland, Department of Chemical and Biomolecular Engineering, College Park, MD, USA

Liquid cell transmission electron microscopy (TEM) electron beam induced growth of nanomaterials has provided many unique insights into nucleation and growth mechanisms of nanoparticles [1-3]. Nanoparticle nucleation and growth occurs via reduction or oxidation of a precursor, typically a dissolved metallic salt, which induces precipitation of solid nanocrystals on the membrane surface of the liquid cell. The reactive species are radicals produced by electron beam induced solvent radiolysis, which for the case of aqueous systems, results in oxidizing hydroxyl and reducing hydrogen radicals [4]. Strongly reducing aqueous electrons also form due to radiolysis. For neat water and electron energies in the range of $100-300 \mathrm{keV}$, approximately equal concentrations of oxidizing and reducing radicals are formed [5]. Classical radiation chemistry experiments employ radical scavengers to eliminate either reducing or oxidizing species and simplify the reaction kinetics to enable quantitative modeling [6]. However, most liquid cell TEM experiments utilizing electron beam induced growth do not employ radical scavengers, so nanoparticle growth (or dissolution) for a given material system often occurs fortuitously, without any attention paid to the reaction pathway leading to growth. Thus, in most cases there are numerous competing reactions, where the reaction with the highest rate of production wins out. Ideally for a specific application, reaction kinetics would be preemptively controlled to achieve growth of the desired product and quantitative analysis of the reaction kinetics [7].

In this talk, I will highlight our recent experiments using radical scavengers to controllably synthesize electrocatalyst nanoparticles on substrates. Transition metals are often employed as alloying components for platinum group metal electrocatalysts, as they induce a favorable d-band shift that reduces the binding strength of reaction intermediates during the oxygen reduction reaction [8]. However, the standard reduction potential of most transition metals (i.e. cobalt, nickel, iron) is negative, indicating they are not easily reduced using radiolytic techniques [9]. For instance, previous experiments have shown aqueous iron [10] and lead [11] ions to be stable under electron beam irradiation. Thus it is necessary to eliminate oxidation reactions during radiolysis to reduce transition metal ions into metallic nanocrystals. We demonstrate the utility of isopropanol and formate anions to eliminate hydroxyl radicals and enable the growth of nickel nanocrystals in situ. Figure 1 shows a representative reaction scheme for the radiolytic reduction of nickel (similar for other transition metals) in the presence of the formate anions as hydroxyl radical scavengers. Without the formate anions, nickel ions are reoxidized by the hydroxyl radicals and no nanocrystal growth occurs. The formate anions selectively convert the oxidizing radicals into reducing carbonate radicals and enable the growth of nickel nanocrystals.

I will also discuss experiments involving electron beam induced growth of bimetallic nanoparticles by co-reducing two metal precursors. In this case, an enhancement factor exists due to the different nanocrystal growth reaction rates of the metal precursors, which can be determined empirically. These 
experiments involve growth experiments of each constituent metal in the bimetallic structure to determine reaction rates, as well as empirical measurement of bimetallic composition using energy dispersive $\mathrm{x}$-ray spectroscopy. The in situ methods presented here should be generally applicable to any metal precursor that is difficult to reduce using electron beam induced growth and should find particular applications for synthesizing bimetallic electrocatalysts for operando TEM experiments [12].

References:

[1] H.M. Zheng, R.K. Smith, Y.W. Jun, et al., Science, 324 (2009), pp. 1309-1312.

[2] Y. Liu, K. Tai, S.J. Dillon, Chemistry of Materials, 25 (2013), pp. 2927-2933.

[3] T.J. Woehl, J.E. Evans, L. Arslan, et al., Acs Nano, 6 (2012), pp. 8599-8610.

[4] Farhataziz, M.A.J. Rodgers, Radiation Chemistry: Principles and Applications, VCH, New York, 1987.

[5] N.M. Schneider, M.M. Norton, B.J. Mendel, et al., Journal of Physical Chemistry C, 118 (2014), pp. 22373-22382.

[6] G.V. Buxton in "Radiation Chemistry: Principles and Applications," ed. M.M. Mostafavi, T. Douki, J. Belloni, EDP Sciences, Orsay, 2008.

[7] T.J. Woehl and P. Abellan, Journal of Microscopy, 265 (2016), pp. 135-147.

[8] J.K. Norskov, J. Rossmeisl, A. Logadottir, et al., Journal of Physical Chemistry B, 108 (2004), pp. 17886-17892.

[9] N. Keghouche, S. Chettibi, F. Latreche, et al., Radiation Physics and Chemistry, 74 (2005), pp. 185-200.

[10] S. Kashyap, T.J. Woehl, X.P. Liu, et al., Acs Nano, 8 (2014), pp. 9097-9106.

[11] E.R. White, S.B. Singer, V. Augustyn, et al., Acs Nano, 6 (2012), pp. 6308-6317.

[12] The author acknowledges the University of Maryland for funding.

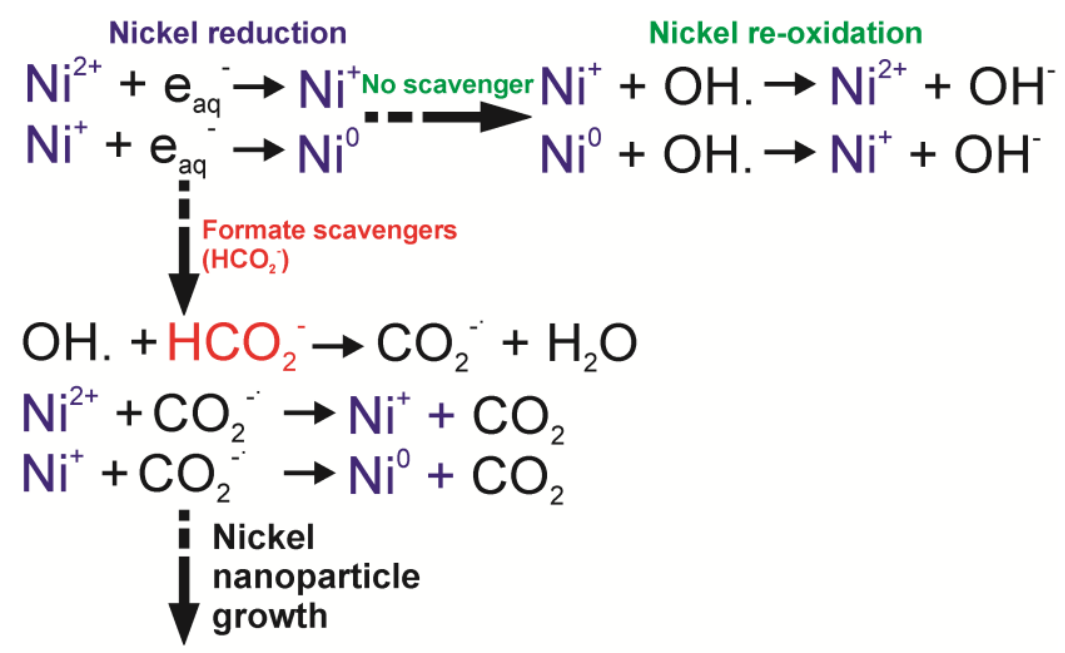

Figure 1. Proposed reaction scheme for radiolytic reduction of aqueous nickel precursor into nickel nanoparticles in the presence and absence of formate anion radical scavengers. 